\title{
Nuclear factor I-A regulates diverse reactive astrocyte responses after CNS injury
}

\author{
Dylan Laug, ${ }^{1,2}$ Teng-Wei Huang, ${ }^{1}$ Navish A. Bosquez Huerta, ${ }^{1,2}$ Anna Yu-Szu Huang, ${ }^{1,2}$ Debosmita Sardar, Joshua Ortiz-Guzman, ${ }^{2,3}$ \\ Jeffrey C. Carlson, ${ }^{1,2}$ Benjamin R. Arenkiel, ${ }^{2,3,4,5}$ Chay T. Kuo, ${ }^{6}$ Carrie A. Mohila, ${ }^{7}$ Stacey M. Glasgow, ${ }^{8}$ Hyun Kyoung Lee, ${ }^{2,3,4,9}$ \\ and Benjamin Deneen ${ }^{1,2,3,4,10}$ \\ 'Center for Cell and Gene Therapy and ${ }^{2}$ Program in Developmental Biology, Baylor College of Medicine, Houston, Texas, USA. ${ }^{3}$ Neurological Research Institute, Texas Children's Hospital, Houston, Texas, USA. \\ ${ }^{4}$ Department of Neuroscience and ${ }^{5}$ Department of Human and Molecular Genetics Baylor College of Medicine, Houston, Texas, USA. ${ }^{6}$ Department of Cell Biology, Duke University, Durham, North Carolina, \\ USA. Department of Pathology, Texas Children's Hospital, Houston, Texas, USA. ${ }^{8}$ Department of Neurobiology, UCSD, San Diego, California, USA. 'Department of Pediatrics, Division of Neurology, Texas \\ Children's Hospital, Houston, Texas, USA. ${ }^{10}$ Department of Neurosurgery, Baylor College of Medicine, Houston, Texas, USA.
}

\begin{abstract}
Reactive astrocytes are associated with every form of neurological injury. Despite their ubiquity, the molecular mechanisms controlling their production and diverse functions remain poorly defined. Because many features of astrocyte development are recapitulated in reactive astrocytes, we investigated the role of nuclear factor I-A (NFIA), a key transcriptional regulator of astrocyte development whose contributions to reactive astrocytes remain undefined. Here, we show that NFIA is highly expressed in reactive astrocytes in human neurological injury and identify unique roles across distinct injury states and regions of the CNS. In the spinal cord, after white matter injury (WMI), NFIA-deficient astrocytes exhibit defects in bloodbrain barrier remodeling, which are correlated with the suppression of timely remyelination. In the cortex, after ischemic stroke, NFIA is required for the production of reactive astrocytes from the subventricular zone (SVZ). Mechanistically, NFIA directly regulates the expression of thrombospondin 4 (Thbs4) in the SVZ, revealing a key transcriptional node regulating reactive astrogenesis. Together, these studies uncover critical roles for NFIA in reactive astrocytes and illustrate how regionand injury-specific factors dictate the spectrum of reactive astrocyte responses.
\end{abstract}

\section{Introduction}

Reactive astrocytes are associated with most forms of neurological disorders, ranging from acute injury to degeneration (1), and play diverse roles in these disease states. They are generally viewed as beneficial during the acute injury response and deleterious during chronic or later stages of recovery (2-7). These paradigms were initially established in spinal cord injury models, however, recent studies on astrocyte diversity have illustrated immense regional and local diversity in the adult brain suggesting that these disparate roles may be the result of a vast reservoir of reactive astrocyte populations $(4,8)$. When this cellular and regional complexity is met with the wide spectrum of disease states that elicit reactive astrocyte responses (7), a complex interplay between diverse resident astrocytes and disease-specific factors emerges. Therefore, deciphering how diverse reactive astrocyte responses are regulated is critical for understanding their contributions to neurological disease.

Despite their ubiquity, the molecular processes that oversee the production of reactive astrocytes after injury remain poorly defined. Critically, reactive astrocytes display a number of features that call to mind their developmental origins, including glial fibrillary acidic protein (GFAP) upregulation, increased proliferative

Authorship note: DL and TWH contributed equally to this work. Conflict of interest: The authors have declared that no conflict of interest exists. Copyright: () 2019, American Society for Clinical Investigation. Submitted: January 16, 2019; Accepted: July 16, 2019; Published: September 9, 2019. Reference information: J Clin Invest. 2019;129(10):4408-4418. https://doi.org/10.1172/JCI127492. capacity, and hypertrophy $(9,10)$. While these are generic features of all reactive astrocytes, using them as molecular entry points and drawing connections to their developmental origins can provide valuable insight into their diverse, post-injury functions (11). For example, STAT3 plays a key role in the generation of astrocytes during development as well as several diverse roles in reactive astrocytes. Conditional deletion of STAT3 in astrocytes impairs glial scar formation after spinal cord injury (SCI), indicating a role for STAT3 in their proliferation or migration (12). Separately, in white matter injury (WMI) models, conditional deletion of STAT3 suppressed remyelination via non-cell-autonomous mechanisms through the promotion of TGF- $\beta 1$ expression in microglia (13). These observations highlight the critical contributions of factors that regulate astrocyte development to reactive astrocyte responses after injury. Moreover, they point to the diverse roles of these factors across a host of neurological disease states, suggesting that developmental factors may be a key to understanding the underlying functional diversity of reactive astrocytes.

Nuclear factor I-A (NFIA) is a transcription factor that plays a central role in astrocyte development, where it is required for the initiation of gliogenesis and the differentiation of astrocytes by direct regulation of key genes essential for astrocyte identity (14, 15). Additionally, we found that NFIA plays a key role in several neurological diseases including glioma and WMI (16-18). Studies of NFIA in WMI focused on its expression in oligodendrocyte precursor populations, finding that its ectopic expression is sufficient to suppress remyelination (18). However, whether NFIA is necessary for remyelination and the cellular origins of this 


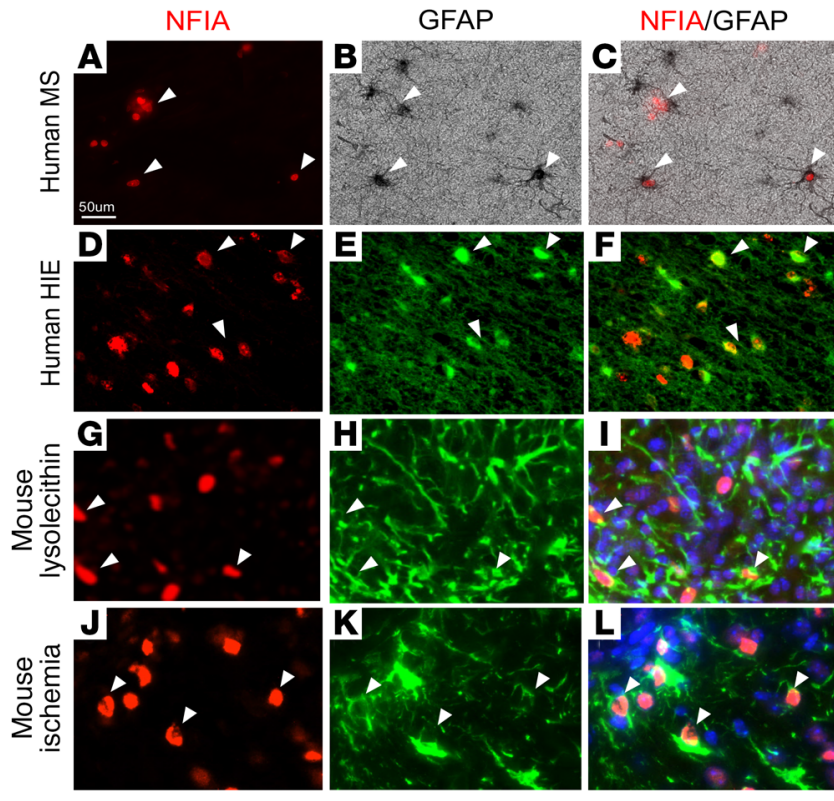

Figure 1. NFIA is expressed in reactive astrocytes. (A-L) Immunofluorescence staining for NFIA and immunohistochemical staining for GFAP in a human MS lesion (A-C), human HIE tissue (D-F), a mouse lysolecithininduced white matter lesion (G-I), and in a mouse photothrombotic ischemia model (J-L). Arrowheads indicate colocalization of NFIA with GFAP. Images of human tissue samples in $\mathbf{A}-\mathbf{F}$ are representative of 3 patients and 8 sections per patient. Images of tissue from the mouse models in $\mathbf{G}-\mathbf{L}$ are representative of 5 animals and 8 sections per animal. Scale bar: $50 \mu \mathrm{m}$.

function remains undefined. Critically, although NFIA is transiently expressed in oligodendrocyte precursors, it continues to be expressed in mature astrocytes in the adult brain $(14-16,18)$. These observations, coupled with the essential role of NFIA in astrocyte development, suggest that this transcription factor may contribute to CNS injury responses through reactive astrocytes. Currently, whether and how NFIA regulates reactive astrocyte responses after CNS injury are unknown.

To initiate studies of NFIA in reactive astrocytes, we evaluated its expression in human adult WMI and neonatal ischemic stroke and found that it was highly expressed in reactive astrocytes in both of these injury states. Combining conditional mouse genetics with spinal cord WMI models and cortical ischemic stroke models, we found that NFIA plays region- and injury-specific roles in reactive astrocytes. In the spinal cord, after WMI, NFIA-deficient astrocytes exhibited defects in blood-brain barrier (BBB) remodeling, which is correlated with the suppression of timely remyelination. In the cortex, after ischemic stroke, we found that NFIA was required for the production of reactive astrocytes from the subventricular zone (SVZ). Mechanistically, we observed that NFIA directly regulated the expression of thrombospondin 4 (Thbs4) in the SVZ, illustrating a key transcriptional node that oversees reactive astrogenesis after stroke. Together, these studies provide the initial characterization of NFIA function in reactive astrocytes after injury and show that it executes distinct functions that are both region and injury dependent. Taken more broadly, these diverse functions of a single transcription factor reinforce the importance of decoding the complex cellular and injury interplay that dictates the spectrum of reactive astrocyte responses.

\section{Results}

NFIA is expressed in reactive astrocytes in human WMI. Our previous studies on NFIA demonstrated its expression in oligodendrocyte precursor (OLP) populations that occupy human multiple sclerosis (MS) and hypoxic-ischemic encephalopathy (HIE) lesions (18). To determine whether NFIA is also expressed in reactive astrocytes present in lesions found in these diseases, we performed doubleimmunofluorescence staining for NFIA and GFAP, a defining marker of reactive astrocytes (1). Using paraffin-embedded human brain tissue obtained from the Rocky Mountain MS Center at the University of Colorado (MS lesions) and Texas Children's Hospital (HIE lesions), we found that NFIA was highly expressed in reactive astrocytes occupying these lesions (Figure $1, \mathrm{~A}-\mathrm{F}$ ). Whereas NFIA showed robust expression in reactive astrocytes, not every GFAP-expressing cell had NFIA expression, suggesting that its expression may be restricted to subsets of reactive astrocyte populations. Moreover, consistent with its expression in OLP populations, we also found that NFIA expression was not limited to GFAP-expressing populations.

To initiate injury modeling studies, we first evaluated NFIA expression in reactive astrocyte populations found in 2 mouse injury models that parallel the human disease states described above. To model remyelination after WMI, we used lysolecithin lesioning of the spinal cord, and to model ischemic stroke, we used cortical photothrombotic stroke $(19,20)$ (Supplemental Figure 1; supplemental material available online with this article; https://doi.org/10.1172/JCI127492DS1). Critically, both models produced a robust reactive astrocyte response, and using double-immunofluorescence, we found that NFIA colocalized with GFAP-expressing reactive astrocytes in these models (Figure 1, G-L). Subsequent analysis of normal, nonreactive astrocytes in uninjured regions of the human and mouse cortex revealed that NFIA was also expressed in resident astrocytes in the healthy brain, suggesting that its expression was not an injury-specific phenomenon (Supplemental Figure 2). Together, these data from primary human samples and relevant animal models indicate that NFIA is expressed in reactive astrocytes associated with WMI and ischemia.

Loss of NFIA in astrocytes delays remyelination after WMI in the spinal cord. To examine whether NFIA contributes to disease pathogenesis through a prospective function in reactive astrocytes, we generated floxed NFIA alleles and intercrossed this line with GFAP-CreER or Sox10-Cre, generating the following lines: NFIA ${ }^{f / f l}$ GFAP-CreER and NFIA ${ }^{f / f l}$ Sox10-Cre. These lines allowed us to selectively delete NFIA in either OLP populations (Sox10Cre) or adult astrocyte populations (GFAP-CreER) and offered the opportunity to resolve its cellular site of action. To determine the role of NFIA in remyelination after WMI, we performed lysolecithin lesioning in the spinal cords of mice from these lines and evaluated the cellular responses 10 days post lesioning (10 dpl), a critical time point that corresponds to the commencement of remyelination in the spinal cord $(21,22)$. As shown in Figure 2, A-J, deletion of NFIA in OLPs (i.e., NFIA fl/fl Sox10-Cre) did not influence the generation of OLPs or GFAP-expressing reactive astrocytes, or have any effect on the kinetics of OLP differentiation into oligodendrocytes during remyelination. These data indicate that loss of NFIA in OLPs does not influence remyelination after WMI. 

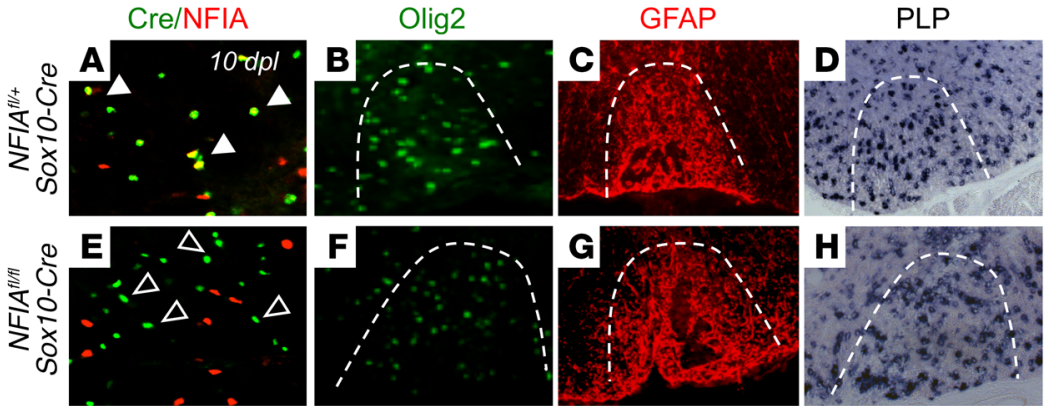

K $N F I A^{i / /+}$

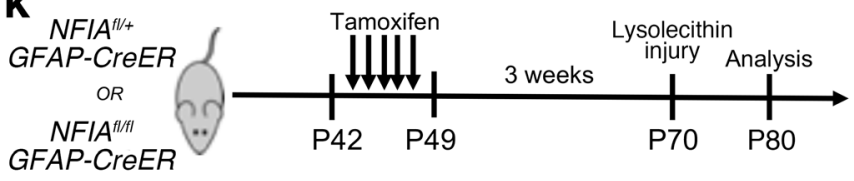

NFIA
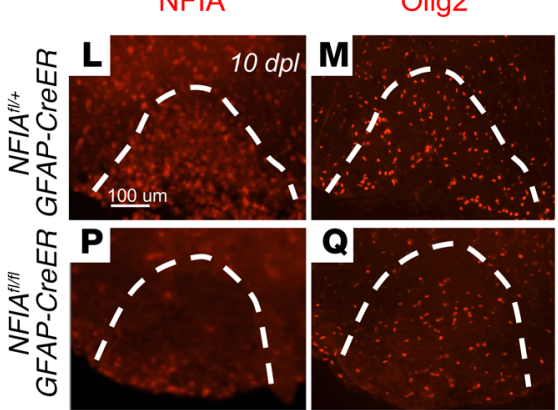

GFAP

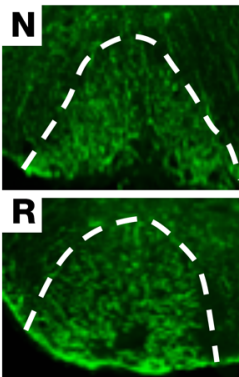

PLP

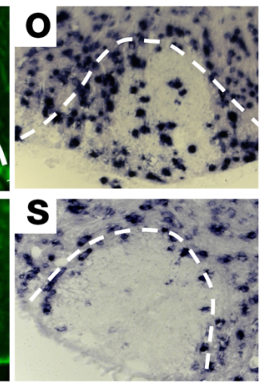

I

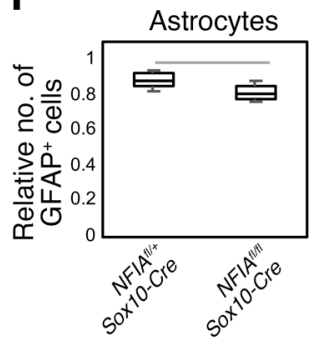

J

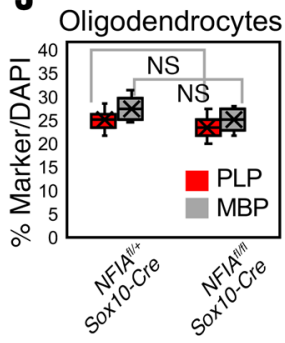

$\mathbf{T}$

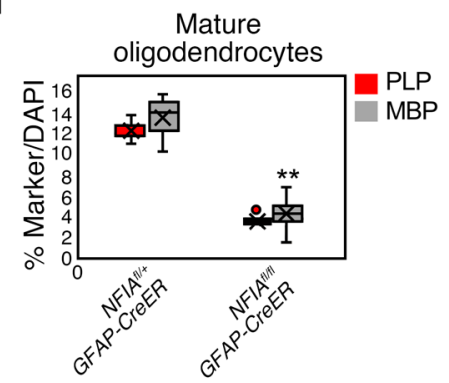

$\mathbf{U}$

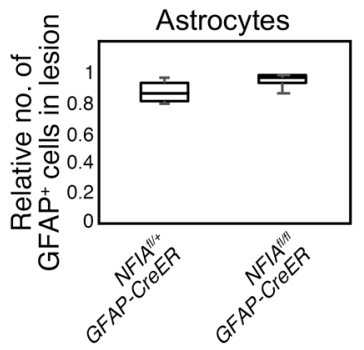

Figure 2. Loss of NFIA from astrocytes delays oligodendrocyte differentiation after WMI. (A-H) Loss of NFIA from the oligodendrocyte lineage did not affect the number of Olig2-expressing cells (B and $\mathbf{F}$ ), GFAP-expressing cells (C, $\mathbf{G}$, and $\mathbf{~ I ) , ~ o r ~ P L P - e x p r e s s i n g ~ c e l l s ~ ( D , ~} \mathbf{H}$, and $\mathbf{J})$ within the lesion after injury (10 dpl). Solid white arrowheads in A indicate Sox10- and NFIA-coexpressing OLP populations; white outlined arrowheads in $\mathbf{E}$ point to Sox $10^{+}$cells without NFIA expression in NFIA ${ }^{f / f l}$ Sox10-Cre mice. (K) Schematic overview of the GFAP-CreERT2 induction schedule and the subsequent WMI time course. (L-S) Induced deletion of NFIA in NFIA ${ }^{f l / f l}$ GFAP-CreER mice effectively eliminated NFIA expression in the lesion (L versus $\mathbf{P}$ ) and did not affect the number of Olig2- or GFAP-expressing cells within the lesion ( $\mathbf{Q}$ and $\mathbf{R}$ ), but resulted in decreased numbers of PLP-expressing cells within the injury site (S). Dashed lines in $\mathbf{A}-\mathbf{H}$ and $\mathbf{L}-\mathbf{S}$ indicate lesion boundaries. ( $R$ and $\mathbf{S}$ ) Quantification of reactive astrocytes (GFAP) and oligodendrocyte marker expression (MBP and $\mathrm{PLP}$ ) in astrocyte-specific NFIA-KO studies. For the analyses in $\mathbf{I}, \mathbf{J}$, and $\mathbf{T}-\mathbf{V}$, the experiments were performed using 4 animals per genotype, and quantification involved 8 sections per animal. Data are shown with box-and-whisker plots. The bounds of the boxes represent upper and lower quartiles, the lines in the boxes represent the median, and the whiskers represent the maximum and minimum values. Data were analyzed by Student's $t$ test. PLP: ${ }^{*} P=$ $1.87 \times 10^{-9}(\mathrm{PLP})$ and ${ }^{* *} P=9.32 \times 10^{-6}(\mathrm{MBP})$. See the Supplemental materials for MBP ISH (Supplemental Figure 3). Scale bar: $100 \mu \mathrm{m}(\mathbf{A}-\mathbf{H}$ and L-S).

To determine whether NFIA functions through astrocytes, we performed lysolecithin lesioning in NFIA ${ }^{f / f l}$ GFAP-CreER mice. Use of the tamoxifen-inducible GFAP-CreER allowed us to bypass any developmental requirements for NFIA in astrocytes or their precursors and enabled us to focus on adult astrocyte populations after injury. As shown in Figure $2 \mathrm{~K}$, we treated mice with tamoxifen starting at 6 weeks of age and then performed lysolecithin lesioning 3 weeks later. Analysis of the cellular responses during early remyelination (i.e., $10 \mathrm{dpl}$ ) revealed efficient deletion of NFIA (Figure 2, L and P), coupled with no overt changes in the number of OLPs marked by oligodendrocyte transcription factor 2 (Olig2) or of reactive astrocytes marked by GFAP (Figure 2, $\mathrm{M}$ and $\mathrm{N}$ versus Figure 2, Q and R; Figure 2, U and V). Next, we evaluated the extent of oligodendrocyte differentiation and found a significant decrease in the number of cells expressing proteolipid protein (PLP) and myelin basic protein (MBP) within the lesions of NFIA $A^{f / f l}$ GFAP-CreER mice (Figure 2, O-T, and Supplemental Figure 3).
Importantly, these changes in oligodendrocyte differentiation are not secondary to axonal loss, as axonal integrity is not differentially affected after injury in these mouse lines (Supplemental Figure 3). Subsequent analysis at $12 \mathrm{dpl}$ (2 days later) revealed that lesions in the NFIA ${ }^{f l / f l}$ GFAP-CreER mice contained PLP- and MBP-expressing cells, indicating a delay in the onset of OLP differentiation (Supplemental Figure 3). Together, these data indicate that loss of NFIA in astrocytes does not influence the generation of OLPs or reactive astrocytes, but rather influences the kinetics of oligodendrocyte differentiation after WMI in the spinal cord.

NFIA-deficient reactive astrocytes demonstrate a defective $B B B$ after WMI. These effects on oligodendrocyte differentiation via NFIA deletion in astrocytes are probably mediated via non-cellautonomous mechanisms. Given that reactive astrocytes are still present in these lesions, we hypothesized that reactive astrocyte function in the absence of NFIA was impaired. One key function of reactive astrocytes is to reestablish the $\mathrm{BBB}$ after injury; 

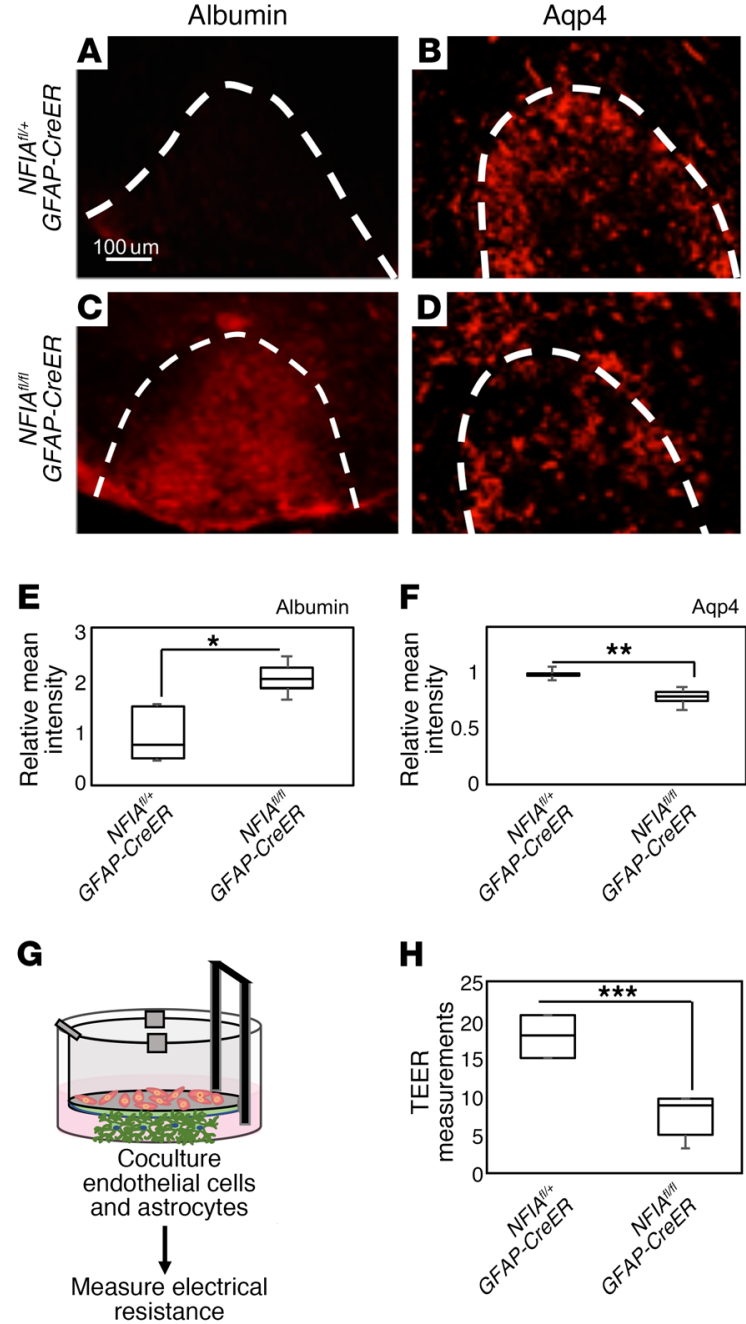

Figure 3. NFIA-deficient astrocytes exhibit impaired BBB remodeling after WMI. (A-D) Deletion of NFIA from astrocytes resulted in an increase in the presence of albumin (A versus $\mathbf{C}$ ) and a decrease in the expression of the astrocyte endfeet protein Aqp4 (B versus $\mathbf{D}$.) Graphs in $\mathbf{E}$ and $\mathbf{F}$ are derived from 4 animals per genotype, and quantification involved 8 sections per animal. (G) Schematic overview of in vitro endothelial/astrocyte barrier assay. (H) NFIA-deficient astrocytes exhibited decreased TEER electrical resistance when cocultured with endothelial cells. The experiment was conducted in triplicate, with 3 coculture wells per genotype for each replicate. Data were analyzed using a Student's $t$ test and are shown as box-and-whisker plots. The bounds of the boxes represent upper and lower quartiles. The lines in the boxes represent the median, and the whiskers represent the maximum and minimal values. ${ }^{*} P=0.01,{ }^{* *} P=0.003$, and ${ }^{* *} P=0.0036$. Scale bar: $100 \mu \mathrm{m}$.

moreover, it has previously been shown that an impaired BBB can suppress oligodendrocyte differentiation after WMI (23). To determine whether the BBB was impaired in NFIA ${ }^{f / f l}$ GFAPCreER lesions, we stained for blood proteins associated with $\mathrm{BBB}$ leakage (albumin) and found very high levels in lesions from NFIA ${ }^{f l / f l}$ GFAP-CreER mice (Figure 3, A versus C and E). This suggested impaired BBB remodeling after WMI. Given the role of astrocyte endfeet proteins in establishing the BBB (24), we evaluated the expression of aquaporin 4 (Aqp4) and found that its expression was also reduced in the absence of NFIA (Figure $3, \mathrm{~B}$ versus $\mathrm{D}$ and $\mathrm{F}$ ). To determine whether these defects in
$\mathrm{BBB}$ integrity were specific to injury conditions or a general feature of this mouse line, we performed the Evans blue assay on uninjured NFIA ${ }^{f / f l}$ GFAP-CreER and control mice and observed no differences in the amount of Evans blue dye extracted from both the spinal cord and cortex of these mice (Supplemental Figure 5). Together, these data suggest that the impaired BBB observed in the presence of NFIA-deficient astrocytes is an injury-specific phenomenon.

To directly examine whether astrocytes lacking NFIA influence the formation of tight junctions between endothelial cells, we performed coculture assays and evaluated the transendothelial electrical resistance (TEER) across this bilayer, an established metric of endothelial integrity (25). To this end, we established astrocyte cultures from NFIA $A^{f / f l}$ GFAP-CreER mice and added tamoxifen to induce deletion of NFIA. Two weeks after tamoxifen-induced deletion of NFIA, we cocultured these astrocytes with brain endothelial cells (bEnd3) (Figure 3G). Upon establishment of the coculture, we measured TEER across this cell bilayer to assay the extent of ion passage across the tight junctions that form between endothelial cells and observed decreased electrical resistance across this cell bilayer in the presence of NFIAdeficient astrocytes (Figure $3 \mathrm{H}$ ). This decrease in TEER indicates that fewer tight junctions were formed between endothelial cells in the presence of NFIA-deficient astrocytes. These data, in conjunction with our analysis of the lesions, indicate that loss of NFIA in reactive astrocytes results in impaired BBB integrity after WMI in the spinal cord.

NFIA is required for the production of reactive astrocytes after cortical ischemic stroke. The results described above implicate NFIA as a key mediator of $\mathrm{BBB}$ remodeling in reactive astrocytes after WMI. To further evaluate this function of NFIA in reactive astrocytes, we extended our studies to the photothrombotic model of cortical ischemia, in which thrombosis results in disruption of endothelial cell integrity (Supplemental Figure 1). It is important to note that NFIA is also highly expressed in reactive astrocytes in this model and in human ischemic disease states (Figure 1), providing a further rationale for these studies. As in our WMI studies, we used the NFIA ${ }^{f l / f l} G F A P$-CreER mice (and NFIA ${ }^{f l /+}$ GFAP-CreER controls) and a defined tamoxifen schedule that allowed us to delete NFIA prior to photothrombotic stroke injury (Figure 4A). Analysis of cellular outcomes 12 days post injury (dpi) revealed efficient deletion of NFIA at the stroke site (Figure 4, C and F), coupled with a dramatic reduction in the number of GFAP-expressing reactive astrocytes (Figure 4, B versus $\mathrm{E}$ and $\mathrm{N}$ ). This reduction in $\mathrm{GFAP}^{+}$reactive astrocytes occurred at the injury border and adjacent to the injury site (Figure 4, H and K). Furthermore, we observed an increased presence of blood proteins (albumin) at the injury site (Figure 4, D versus $G$ ), which was consistent with a reduced production of reactive astrocytes and the associated impaired injury response. These observations suggested a depletion of resident astrocytes or an inability of resident astrocytes to acquire reactive properties. To decipher between these possibilities, we stained the injury border and adjacent regions with Sox9, an established marker of mature astrocytes in the brain (26). As shown in Figure 4O, there was no change in the number of Sox9-expressing cells at the injury site (Figure 4, I and J versus $\mathrm{L}$ and $\mathrm{M}$ ), nor in adjacent regions, indicating that loss of NFIA was not influencing the survival or local 
A
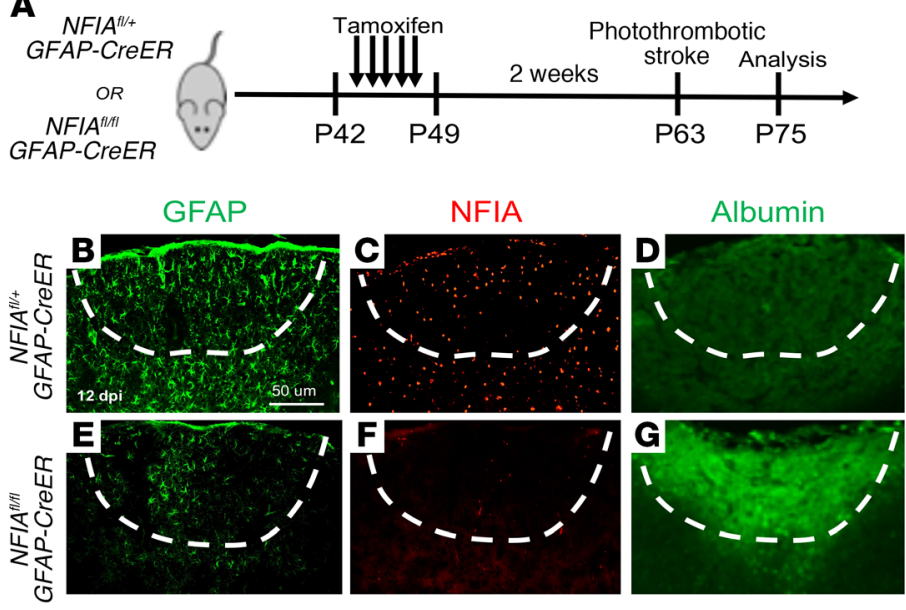

GFAP
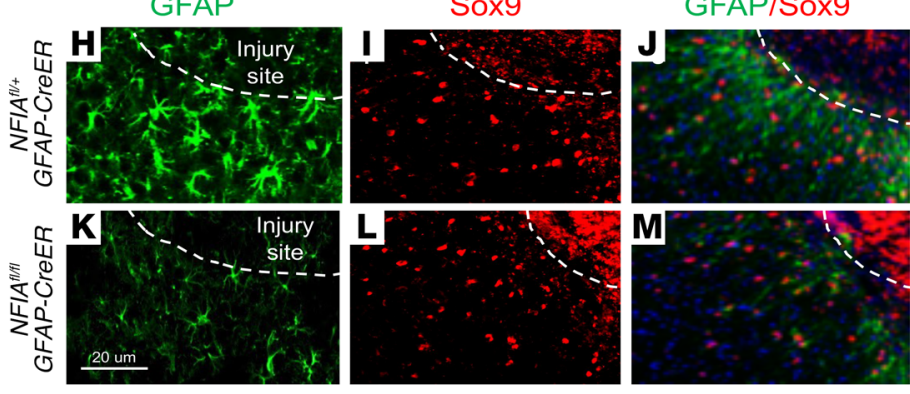

Figure 4. NFIA is required for the generation of reactive astrocytes after ischemic stroke. (A) Overview of the experimental procedure for GFAP-CreER induction and the stroke injury model. (B-G) Loss of NFIA in astrocytes resulted in a reduction of GFAP' reactive astrocytes (B versus $\mathbf{E}$ ) and an increase in the blood serum protein albumin (D versus $\mathbf{G}$ ) at the stroke injury site (12 dpi). Dashed lines in B-I indicate the injury boundaries. (H-M) High-magnification images of GFAP ${ }^{+}$and Sox9+ cells at the injury site in control and NFIA ${ }^{\text {flff }}$ GFAP-CreER mice, indicating a decrease in the number of GFAP ${ }^{+}$reactive astrocytes at the injury site, coupled with no change in the number of Sox9+ cells. Quantification of GFAP' reactive astrocytes $(\mathbf{N})$ and Sox9-expressing cells $(\mathbf{0})$ in response to the photothrombotic stroke model in control and NFIA fl/fl GFAP-CreER mice. Quantification data were derived from 4 animals per genotype for $\mathbf{N}$ and 3 animals per genotype for $\mathbf{0}$ and 8 sections per animal in all cases. ${ }^{*} P=0.0092$, by Student's $t$ test. Data are shown as box-and-whisker plots. The bounds of the boxes represent upper and lower quartiles, the lines in the boxes represent the median, and the whiskers represent the maximum and minimum values. Scale bars: $50 \mu \mathrm{m}$ (B-G) and $20 \mu \mathrm{m}(\mathbf{H}-\mathbf{M})$.

proliferation of astrocytes in the cortex after ischemic stroke injury. Together, these data suggest that NFIA expression is necessary for local astrocytes to acquire reactive properties after ischemic stroke in the cortex and, together with our spinal cord WMI studies, indicate that this transcription factor has region- and/or injury-specific functions in reactive astrocytes.

To ascertain whether NFIA demonstrates region-specific functions in reactive astrocytes, we performed lysolecithin lesioning in the corpus callosum of the adult brain, using the NFIA ${ }^{f l / f l}$ GFAPCreER mouse lines and the tamoxifen induction paradigm shown in Figure 2K. Oligodendrocyte regeneration was evaluated $12 \mathrm{dpl}$ of the corpus callosum, a relatively early time point in the myelin repair process for this region (27). Our analysis revealed no changes in the extent of oligodendrocyte differentiation or in the production of reactive astrocytes in these lesions (Supplemental Figure 4), indicating that loss of NFIA does not influence repair in this context. These data, in conjunction with our spinal cord and ischemic stroke studies, indicate that the role of NFIA in reactive astrocyte responses varies across both the injury type and region of the CNS.
N

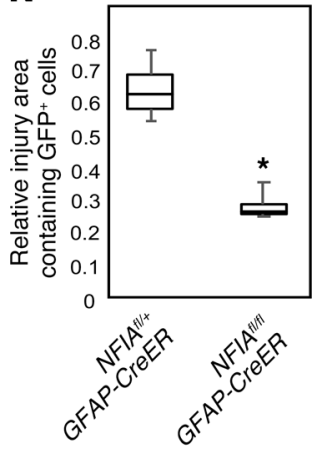

0

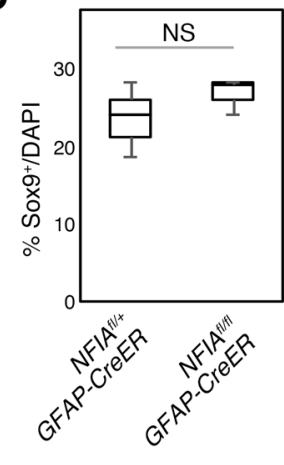

Reactive astrogenesis in the SVZ is impaired in the absence of NFIA. To understand the cellular mechanism by which NFIA controls the generation of reactive astrocytes after cortical ischemia, we focused on reactive astrogenesis from the SVZ. Previous studies have shown that the SVZ is a source of reactive astrocytes that emigrate to injury sites after cortical ischemia and that loss of SVZ-derived reactive astrocytes dramatically impairs the injury response and recovery $(19,28)$. To determine whether SVZ responses after ischemic injury are also affected by loss of NFIA, we implemented our deletion/injury paradigm (Figure 4A), treated with BrdU 5 dpi, and subsequently harvested cortices from NFIA ${ }^{f l / f l}$ GFAP-CreER and control mice. First, we found that NFIA was highly expressed in BrdU ${ }^{+}$cells occupying the SVZ under these conditions in the control animals (Figure $5, A^{-} \mathrm{C}$ ), indicating that it is expressed in cell populations that are able to generate reactive astrocytes. Next, we evaluated BrdU incorporation into the SVZ in the absence of NFIA and observed efficient deletion of NFIA in this region (Figure 5, A versus D), coupled with a drastic decrease in the extent of cellular proliferation (Figure 5, B and C versus E-G). Interestingly, we also observed decreased BrdU incorporation within the SVZ in the NFIA ${ }^{f l / f l}$ GFAP-CreER mice under noninjury conditions (Supplemental Figure 5), indicating that NFIA plays a general role in maintaining proliferative cell populations in the SVZ under homeostatic conditions. These data indicate that NFIA is required for SVZ proliferation in the uninjured brain and after cortical ischemia, suggesting that defects in reactive astrogenesis could be a result of these defects in the SVZ.

The foregoing data implicate the SVZ as one of the prospective sites of action of NFIA in this post-injury context. To test whether defects in reactive astrogenesis are due to loss of NFIA in the SVZ, we used virus-mediated approaches to selectively delete NFIA in the SVZ. To this end, we generated NFIA ${ }^{f / f l}$ Rosa-flox-tdTomato mouse lines and injected the SVZ of these mice with adenovirus type 5 (Ad5) virus containing Cre (Figure $5 \mathrm{H}$ ). This approach enabled the following: (a) fate-mapping of Ad5-Cre-infected cells in the SVZ after injury and (b) specific deletion of NFIA from SVZ cell populations. Following the injection/injury paradigm depicted in Figure $5 \mathrm{H}$, in control animals, we found a host of tdTomato-labeled, NFIA-expressing cells at the injury site that also coexpressed GFAP (Figure 5, I-L). These observations indicate that a subset of reac- 

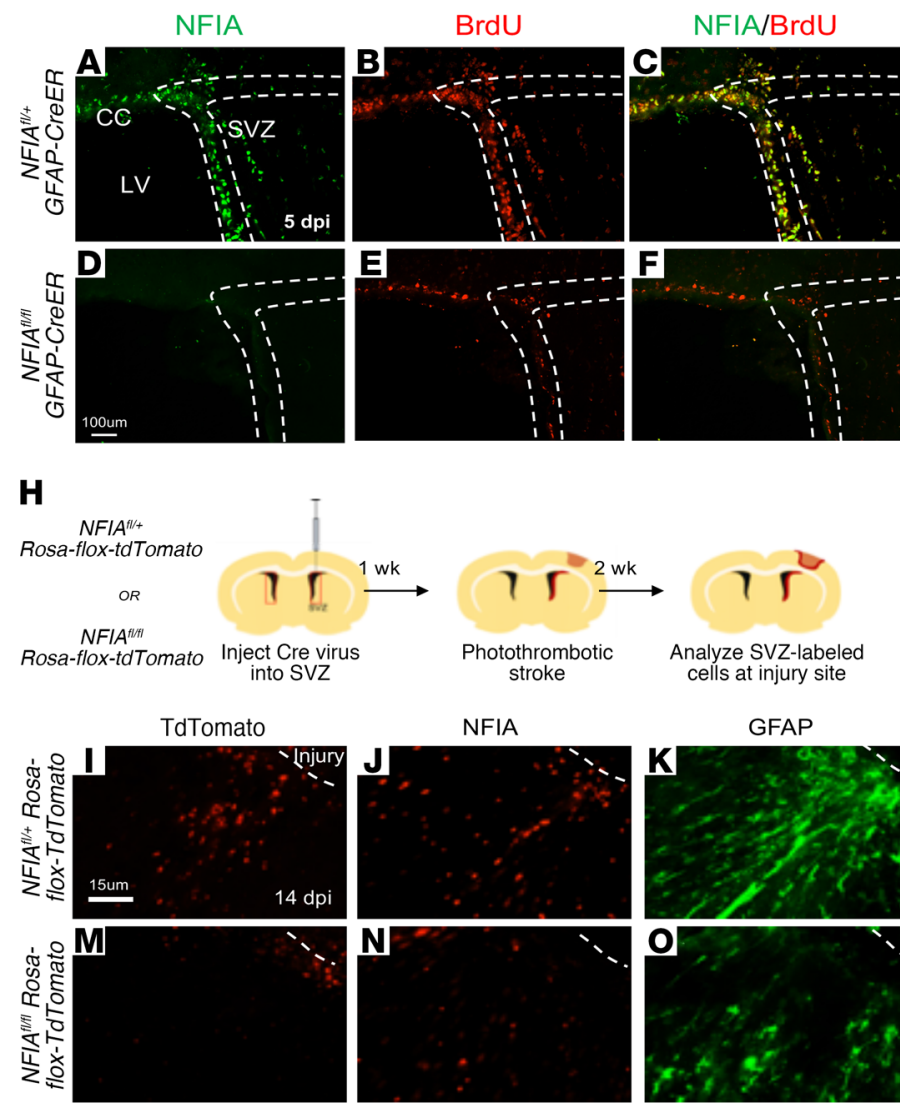

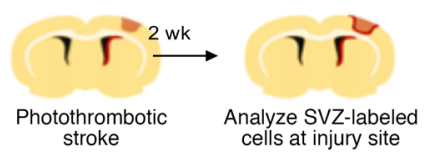

GFAP
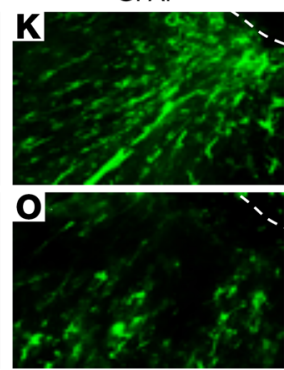

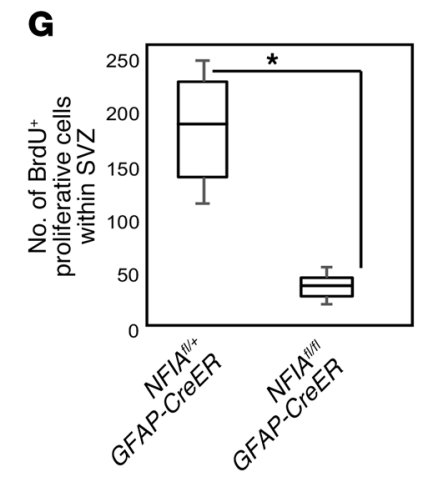

Figure 5. Selective deletion of NFIA in the SVZ impairs reactive astrocyte production after ischemia. (A-C) NFIA was expressed in proliferating BrdU populations in the SVZ at $5 \mathrm{dpi}$, and genetic deletion of NFIA with GFAP-CreER resulted in decreased proliferation, as measured by BrdU incorporation into SVZ cell populations (D-F). (G) Quantification of BrdU+ cells within the SVZ after injury in control and NFIA ${ }^{f l f l}$ CFAP-CreER mice. Quantification was derived from 5 control and 4 experimental animals and 8 sections per animal. ${ }^{*} P=0.0016$, student's $t$ test. (H) Experimental timeline for Cre virusmediated deletion of NFIA from SVZ progenitor cells and subsequent photothrombotic stroke and analysis. (I-L) Control experiments showing SVZderived, tdTomato-labeled cell populations (I), NFIA expression (J), and generated reactive astrocytes residing at the injury site (K and $\mathbf{L})$. (M-P) Deletion of NFIA from SVZ cell populations resulted in decreased numbers of tdTomato ${ }^{+}$cells (M) and reduced expression of NFIA ( $\mathbf{N}$ ) and GFAP (0 and $\mathbf{P}$ ) at the injury site. (Q) Quantification of tdTomato cells at the injury border in NFIA ${ }^{f l /+}$ Rosa-tdTomato and NFIA ${ }^{f l f l}$ Rosa-tdTomato mice. Quantification was derived from 8 sections per animal and 3 animals per genotype. ${ }^{*} P<0.05$ and ${ }^{* *} P=0.0065$, by Student's $t$ test. Data are shown as box-and-whisker plots. The bounds of the boxes represent upper and lower quartiles, the lines in the boxes represent the median, and the whiskers represent the maximum and minimal values. Scale bars: $100 \mu \mathrm{m}(\mathbf{A}-\mathbf{F})$ and $15 \mu \mathrm{m}(\mathbf{I}-\mathbf{P})$. CC, corpus callosum; LV, lateral ventricle.

tive astrocytes at the ischemic injury site were derived from NFIAexpressing cells from the SVZ. Next, we performed the same experiment in the NFIA ${ }^{f / f l}$ Rosa-flox-tdTomato mouse and detected a drastic decrease in the number of tdTomato-labeled cells coexpressing NFIA at the injury site (Figure 5, M, N, and Q), as well as a decrease in the number of GFAP-expressing reactive astrocytes (Figure 5, O and P). Critically, analysis of the SVZ of these mice revealed that a majority of the tdTomato-labeled cells remained in the SVZ, indicating that the cells infected with Ad5-Cre remained in the SVZ and did not mount an injury response (Supplemental Figure 5). These data, in conjunction with our analysis of the post-injury SVZ, indicate that NFIA is required for SVZ astrogenesis after ischemic injury.

NFIA directly regulates Thbs 4 expression. Next, we sought to decipher the mechanism by which NFIA regulates post-ischemic SVZ astrogenesis. Previous studies have shown that Thbs4 is required for SVZ astrogenesis after photothrombotic stroke (19), and our results showed that conditional NFIA KO in astrocytes phenocopied the Thbs $4 \mathrm{KO}$, which suggests a link between these genes. Because NFIA is a transcription factor and Thbs 4 is expressed in the SVZ and immediately adjacent regions (19), we hypothesized that Thbs4 may be a target of NFIA. To investigate this possibility, we first evaluated Thbs4 expression in NFIAdeficient mice and found that its expression was completely eliminated in the SVZ, rostral migratory stream (RMS), and corpus callosum (CC) in the absence of NFIA in the normal brain and after ischemic injury (Figure 6, A-E, and Supplemental Figure 6). Next, we queried the core promoter region of Thbs4, identifying a prospective NFIA-binding site proximal to the transcriptional start site that we confirmed via ChIP with NFIA antibodies on cultured astrocytes (Figure 6, F and G). Finally, we cloned the core promoter of Thbs 4 that contains this NFIA-binding site and found that increasing concentrations of NFIA could effectively activate this promoter region (Figure $6 \mathrm{H}$ ). Together, these genetic and biochemical data indicate that Thbs 4 is a direct transcriptional target of NFIA in the SVZ.

Our collective studies suggest a model by which NFIA regulates Thbs4 expression in the SVZ after ischemic stroke and that this transcriptional node plays a critical role in reactive 


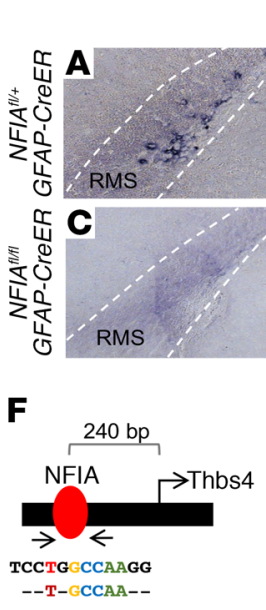

Thbs4
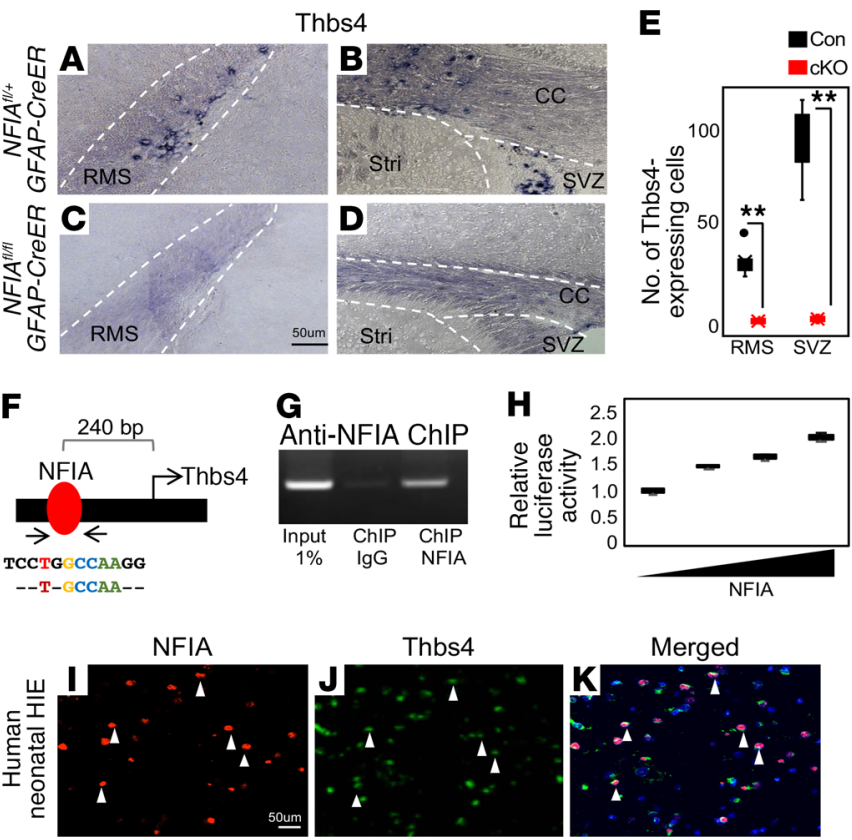

NFIA
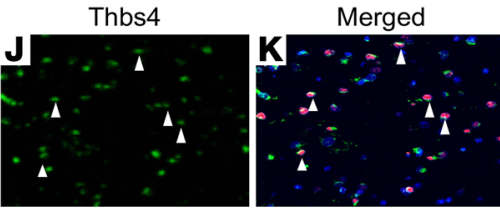

Figure 6. NFIA directly regulates the expression of Thbs4. (A-D) Deletion of NFIA resulted in decreased Thbs4 mRNA expression within the RMS and SVZ regions. (E) Quantification of Thbs4 ISH signal within the RMS and SVZ regions in NFIA ${ }^{f l /+}$ CFAP-CreER versus NFIA ${ }^{f / f l}$ GFAP-CreER mice. Quantification was performed by analyzing 8 sections per animal and 3 mice per genotype. Data are shown with box and whisker plots. The bounds of the boxes represent upper and lower quartiles, the lines in the boxes represent the median, and the whiskers represent the maximum and minimal values. ${ }^{* *} P=0.000053$ (RMS) and ${ }^{* *} P=0.0000026$ (SVZ), by Student's $t$ test. (F) Illustration of the prospective NFIA-binding site 240 bp upstream of the Thbs 4 transcriptional start site and alignment with the consensus NFIA-binding sequence. (G) Anti-IgG and anti-NFIA antibodies were used to immunoprecipitate DNA fragments from cultured astrocytes. Fragments were analyzed via PCR using primers designed to include the prospective NFIA-binding site within the Thbs4 promoter region and showed that NFIA bound this predicted site. (H) Increasing concentrations of NFIA resulted in an increased activation of a luciferase reporter plasmid containing the Thbs4 promoter region when cotransfected in P19 cells. Data are shown as box-and-whisker plots. The bounds of the boxes represent upper and lower quartiles, the lines in the boxes represent the median, and the whiskers represent the maximum and minimal values. (I-K) Fluorescence immunostaining for NFIA combined with fluorescence ISH labeling for Thbs4 indicated coexpression within human neonatal HIE tissue. Solid white arrowheads show colabeling of Thbs4 and NFIA. Images in I-K are representative of tissue samples from 3 individual patients, 8 sections per patient. Scale bars: $50 \mu \mathrm{m}$ (A-D and I-K).

These findings highlight critical parallels between glial development and the glial response after injury. During development, NFIA expression within neural precursor cells of the spinal cord is necessary and sufficient to initiate gliogenesis $(14,15)$. Our data suggest that NFIA is similarly important for reactive astrogenesis within the SVZ after cortical ischemic injury. Interestingly, other factors important for glial development have also been linked to reactive astrocyte responses to injury. A number of studies have implicated the JAK/STAT pathway as being important for glial development through the inhibition of pro-neural transcription factors and induction of glial genes via the JAK/STAT mediator STAT3 (29-32). Similar to the developmental parallels highlighted in our study of NFIA, studies utilizing spinal cord injury models found that the loss of STAT3 impairs reactive astrocyte responses and results in aberrant reactive astrocyte function after injury $(12,13)$. Other examples include the requirement of the Notch signaling receptor Notch1 in both cerebellar glial development and the generation of reactive astrocytes in response to stroke events $(33,34)$. Moreover, Notch signaling in reactive astrocytes can suppress OLP repair after WMI (35). Together, these studies mirror and complement our findings regarding the importance of key regulators of glial development in reactive astrocyte generation and function after CNS insult.

In future studies, it will be important to understand how these developmental factors cooperate in the regulation of reactive astrocyte generation and function. Considering that STAT3 and NFIA have been shown to cooperate in the transcriptional regulation of GFAP expression during developmental gliogenesis (36), there is a possibility that such relationships persist after development, especially in the context of reactive gliosis. Similar mechanistic relationships between NFIA and the omnipotent Notch signaling pathway may also reveal critical developmental processes that are repurposed after injury.

Region- and injury-specific roles for NFIA in reactive astrocytes. By subjecting NFIA conditional mutants to WMI within the spinal 
cord and corpus callosum and to ischemia within the cortex, we have demonstrated that NFIA has distinct functions within astrocyte populations in these different contexts. Among reactive astrocytes of the spinal cord, NFIA is vital for the integrity of the BBB after WMI, but not for the corpus callosum. These data suggest that NFIA plays a region-specific role in reactive astrocyte responses to WMI, highlighting an underlying functional diversity across these regions. These observations are in stark contrast to our findings in models of cortical ischemia, in which NFIA was crucial for both the production of reactive astrocytes via proliferation within the SVZ and the acquisition of reactive characteristics. Collectively, these observed differences in the function of NFIA in reactive astrocytes within the spinal cord versus the cortex, across distinct injury paradigms, provide what we believe to be an important conceptual advance - that a single transcription factor can execute regionand/or injury-specific functions within the same lineage.

This raises the possibility that there are injury- and/or region-specific factors that influence how NFIA operates in reactive astrocytes in order to modulate their generation and function. Because different types of CNS injuries and diseases result in varying amounts of cell death, inflammation, cellular responses, and tissue replacement and remodeling, it is possible that these events result in differential signaling and the release of factors within parenchyma at the injury site that coordinate cellular responses unique to a specific CNS insult (37). Indeed, several studies have begun to characterize the molecular response of reactive astrocytes to different signaling events that occur as a result of injury (38-40).

Also, regional diversity must be taken into account when considering diverse reactive astrocyte responses. Recent studies have shown that different brain regions harbor diverse populations of astrocytes that exhibit both molecular and functional differences $(8,41,42)$. Although the source of this diversity remains largely unknown, it is possible that brain anatomy and the underlying proximity and relationship of astrocytes to other CNS cells and structures are at least partially responsible. In support of this, one study has shown that postnatal astrocytes of the dorsal spinal cord differ significantly in their gene expression profiles from their ventral counterparts (43). Are similar positional cues giving rise to the functional diversity of NFIA in reactive astrocytes, and could these same positional cues also drive diverse functions for NFIA in nonreactive astrocytes? If such regionality exists, it calls to mind patterning mechanisms underlying nervous system development, in which the positional cues direct the differentiation of progenitors into distinct cell populations $(44,45)$. Thus, understanding how patterning mechanisms contribute to astrocyte responses is yet another area of study that may influence CNS injury and associated responses (46). A detailed understanding of the regional diversity of reactive astrocytes as well as how different injury types influence these diverse reactive astrocytes may lead to the development of more selective therapies that will aid in stimulating specific repair programs and result in better patient outcomes.

Transcriptional control of injury-induced SVZ astrogenesis. Our studies also describe a transcriptional link between NFIA and Thbs4 in the SVZ that regulates the generation of reactive astrocytes in response to a cortical ischemic event. Thbs 4 has previously been identified as a key regulator of the SVZ reactive astrocyte response (19). Furthermore, we found that loss of NFIA resulted in a significant decrease in the expression of Thbs 4 within the SVZ (Figure 6). These findings, along with our observations that NFIA directly regulated Thbs 4 expression and that loss of NFIA from the SVZ phenocopied the reduced reactive astrocyte response in Thbs4-KO mice, suggest that the regulation of Thbs 4 by NFIA is an important mediator of the SVZ cellular response to cortical ischemia. Together, our studies reveal what we believe to be a novel role for NFIA in the generation of reactive astrocytes from the SVZ after such cortical injuries.

These data raise the possibility that NFIA may also be involved in the transcriptional control of reactive astrocyte responses from the SVZ in other diseases and injuries. Previous studies have found that the SVZ can generate oligodendrocytes in response to WMI within the corpus callosum and periventricular white matter and that EGFR signaling within the SVZ is important for this response (47-49). These observations, coupled with our data showing that NFIA regulates the proliferation and subsequent generation of reactive astrocyte responses from these same SVZ progenitor populations after injury, raise interesting questions about the role that NFIA may play in mounting an SVZ-proliferative response to any number of injuries within the brain. These dividing cells may then be influenced by injury-specific signaling events, such as EGFR signaling, to generate different proportions of reactive astrocytes or OLPs as required for the repair of that specific injury. More broadly, NFIA has been shown to directly repress the cell-cycle regulator p21 in a mouse neural stem cell model of glioma, suggesting that NFIA may have roles in promoting proliferation outside of the SVZ after injury (50). Several studies have shown that reactive astrocytes exhibit some localized proliferative capacity at CNS injury sites $(34,51)$. Because NFIA is expressed by reactive astrocytes, it is possible that NFIA plays a role in the proliferation that occurs at the injury periphery among these cells.

Our data identify NFIA as a central regulator of SVZ reactive astrocyte production after cortical ischemia through direct transcriptional activation of Thbs4. Thbs 4 has been shown to physically interact with Notch1 to activate downstream effectors of the Notch pathway. Interestingly, a recent study found that NFIA expression increased in primary SVZ-derived neural stem cells after being treated with Thbs4 (19). However, this study found no significant decrease in NFIA expression in Thbs $4 \mathrm{KO}$ animals (19). In conjunction with our data regarding the direct transcriptional regulation of Thbs 4 by NFIA, these findings suggest that there may be positive feedback activation of NFIA through its activation of Thbs 4 transcription. Intriguingly, our data provide genetic evidence that Thbs4 expression is dependent on NFIA in homeostatic, noninjured conditions, suggesting that NFIA operates primarily upstream of Thbs4. Because these previous studies of the relationship of Thbs 4 to Notch and NFIA primarily focused on injury conditions, it is possible that this positive feedback loop only occurs within the SVZ in response to injury states. If true, this would support the hypothesis that NFIA has functional diversity in gene regulation when the CNS is subjected to injury.

\section{Methods}

Genetic KO of NFIA. NFIA ${ }^{f / f l}$ conditional mutant mice were generated using targeted mutation of embryonic stem (ES) cells from 
the European Mouse Mutant Cell Repository (EuMMCR clone ID: HEPD0646_5_E04). ES cells were injected into 3.5 dpc blastocysts, which were then transferred into pseudopregnant mothers, all according to protocols available on the EuMMCR website (https:// www.eummcr.org/). NFIA ${ }^{f l /+}$ mice were then intercrossed to generate $N F I A^{f l / f l}$ animals.

Targeted KO of NFIA was regulated with GFAP-CreERT2 mice (The Jackson Laboratory, stock no. 012849) and Sox10-Cre mice (a gift from Jeff Neul, Vanderbilt University, Nashville, Tennessee, USA).

To induce recombination in the GFAP-CreERT2 mouse line, animals were injected i.p. once a day for 5 consecutive days with a $100-\mathrm{mg} / \mathrm{kg}$ body weight dose of tamoxifen (MilliporeSigma) (10 $\mathrm{mg} / \mathrm{mL}$ dissolved in a 9:1 corn oil/ethanol mixture). This regimen was sufficient to induce recombination in more than $95 \%$ of the $\mathrm{GFAP}^{+}$cells surveyed by NFIA and GFAP immunofluorescence for the GFAP-CreERT2 line.

ISH and IHC. mRNA ISH probes generated for murine myelin PLP were generated, and an ISH protocol was performed as previously described (4). An mRNA probe for murine Thbs4 was generated using primers obtained from the Allen Brain Atlas (forward primer: AGGGAGCAGAAGAGTCACCAT; reverse primer: GACTGAAAGCTGAGAGGACCAC) according to previously published methods (52). Double-ISH/IHC was performed as previously described (14). Briefly, fluorescence ISH for Thbs4 on human HIE tissue was performed first and developed with anti-digoxigenin-POD (Roche) and then fluorescently amplified using the TSA Plus Cy5 Fluorescence System (PerkinElmer). Following ISH, fluorescence staining for NFIA was performed using rabbit anti-NFIA (MilliporeSigma, 1:500) as described below.

For immunohistochemical analysis of this tissue, slides with sections were washed 3 times in $1 \times$ PBS and blocked for 1 hour in $5 \%$ goat serum in PBS with addition of $0.01 \%$ Triton (excluded from the blocking solution used for membrane-bound proteins), followed by incubation with primary antibody dilutions overnight. The following primary antibodies were used: rabbit anti-Olig2 (AB9610, Abcam, 1:1000); rabbit anti-GFAP (Z0334, Dako, 1:1000); mouse anti-GFAP (MAB360, MilliporeSigma, 1:500); rabbit anti-NFIA (HPA006111, MilliporeSigma, 1:500); rabbit anti-Sox9 (Ab5535, MilliporeSigma, 1:1000); rabbit anti-albumin (ab135575, Abcam, 1:100); rabbit anti-Aqp4 (AB2218, MilliporeSigma, 1:250); rat anti-BrdU (ab6326, Abcam, 1:500); mouse anti-Cre (MAB3120, MilliporeSigma, 1:500); chicken anti-neurofilament heavy polypeptide (ab4680, Abcam, 1:1000); mouse anti-nonphosphorylated neurofilament $\mathrm{H}$ (801702, BioLegend, 1:800); and goat anti-Thbs4 (sc-390734, Santa Cruz Biotechnology, 1:150).

Lysolecithin-induced demyelination model. Demyelinated lesions were generated in the ventrolateral white matter of the spinal cord or the corpus callosum of 8- to 10-week-old mice as previously described (11, 16). Briefly, the ventral white matter of the spinal cord was injected with $0.5 \mu \mathrm{L} 1 \%$ lysolecithin (MilliporeSigma) dissolved in sterile $1 \times$ PBS solution using a $10-\mu \mathrm{L}$ Hamilton syringe and the KD Scientific syringe pump system. Mice were harvested at $10 \mathrm{dpl}$ and were transcardially perfused with ice-cold $1 \times$ sterile PBS followed by a $30-\mathrm{mL}$ perfusion of $4 \%$ paraformaldehyde at a rate of $1 \mathrm{~mL} /$ minute using a Thermo Fisher Scientific variable speed minipump. CNS tissues were dissected and fixed in $4 \%$ paraformaldehyde overnight and then cryoprotected in $30 \%$ sucrose for an additional 24 hours. Next, tissue samples were frozen in Tissue-Tek O.C.T. compound embedding medium and sectioned into $20-\mu \mathrm{m}$ slices that were then directly mounted onto glass slides.
Photothrombotic focal ischemia model. Focal ischemic sites were generated in the cortex of 8- to 10 -week-old mice using previously described methods (20). Briefly, mice were injected i.p. with $10 \mu \mathrm{L} / \mathrm{g}$ body weight rose Bengal dye dissolved $(15 \mathrm{mg} / \mathrm{mL})$ in sterile phosphate buffered saline. After waiting 15 minutes for the dye to enter and circulate in the bloodstream, the mouse was anesthetized using isoflurane ( $2 \%$ in $2 \mathrm{~L} / \mathrm{min}$ oxygen), and an incision was made in the skin overlaying the skull. Next, a Leica KL 300 LED light affixed with a custom-made 1-mm-diameter aperture was centered at $0.5 \mathrm{~mm}$ anterior and $1.0 \mathrm{~mm}$ lateral to bregma. The underlying vasculature was exposed to light from this source for 15 minutes, after which the light was turned off, the skin was sutured, and the animal was allowed to recover in sternal recumbency until fully awake. Upon harvesting of the tissue at 12 or $14 \mathrm{dpi}$, the animals were perfused, and the tissue was processed as described above.

Cre virus injection into the SVZ. For all stereotaxic injections, mice were anesthetized using vaporized isoflurane and maintained under anesthesia using $2 \%$ isofluorane $\mathrm{O}_{2}$. All injections were performed using a stereotaxic apparatus synced to Leica Angle Two software for coordinate guidance. For Cre virus-mediated deletion of NFIA, SVZ progenitor cells were bilaterally injected into the SVZ in NFIA ${ }^{\mathrm{f} / \mathrm{fl}}$ Rosa-flox-tdTomato and NFIA ${ }^{\mathrm{A} / \mathrm{+}}$ Rosa-flox-tdTomato mice as follows: anteroposterior $(\mathrm{AP})=$ +0.40 ; dorsoventral $(\mathrm{DV})=-3.40,-3.20,-3.00,-2.80,-2.60,-2.40$; mediolateral $(\mathrm{ML})=-1.00$, with $119 \mathrm{~nL}$ per site of injection along the $\mathrm{DV}$ axis. In total, each hemisphere was injected 6 times with $119 \mathrm{~nL}$ per DV site per hemisphere using Ad5-iCre-GFP virus.

Human WMI acquisition and staining. Adult brain MS tissue was acquired through collaboration with the Rocky Mountain Multiple Sclerosis Society. Attainment of neonatal tissue and its HIE pathological diagnosis were performed by Carrie Mohila of Texas Children's Hospital as previously described (11). IHC on paraffin-embedded tissue was performed as previously described (11). Briefly, after deparaffinization, tissue was subjected to antigen retrieval for 7 minutes in $1 \times$ Na citrate, pH 6.0, followed by 15 minutes of $3 \% \mathrm{H}_{2} \mathrm{O}_{2}$ incubation, blocking, and, finally, primary antibody incubation overnight. The following antibodies were used: anti-NFIA (rabbit, MilliporeSigma) and anti-GFAP (mouse, MilliporeSigma). For colorimetric GFAP staining, slides were washed with PBS and then incubated with ImmPRESS HRP anti-mouse antibody (Vector Laboratories). Colorimetric reaction was done using ImmPACT DAB peroxidase according to the manufacturer's guidelines (Vector Laboratories). For double-immunolabeling with NFIA on this tissue, following colorimetric DAB peroxidase reaction, the slides were washed and then incubated with anti-NFIA antibody for 48 hours. NFIA staining was then visualized with goat anti-mouse Alexa Fluor 568 (Invitrogen, Thermo Fisher Scientific). Slides were counterstained with DAPI and mounted in VECTASHIELD. Slides were then imaged using combined bright-field and fluorescence microscopy.

Luciferase reporter assay. A mouse Thbs 4 promoter ( -1 to -810 relative to the transcription start site) containing the putative NFIA-binding site was amplified by PCR using mouse genomic DNA and subcloned into a pGL3-basic luciferase reporter plasmid (Promega). The putative NFIA-binding site was predicted by sequence conservation between species and contained the following sequence: 5 '-...CAAATGGGGGATCCTGGCCAAGGGGGCC...-3'.

The P19 cell line was cotransfected with a Thbs4 pGL3-reporter construct, a CMV- $\beta$-gal vector, and different amounts of pcDNA3-FlagNFIA using Lipofectamine 2000 Transfection Reagent (Thermo Fisher 
Scientific). $\beta$-Gal was used for normalization of transfection efficiency. Cells were harvested 48 hours after transfection, and luciferase activity was measured using Luciferase Assay Reagent II (LARII) (Promega).

In vitro $B B B$ assay and TEER measurements. Astrocytes were obtained from the cortices of P0 to P1 NFIA ${ }^{f /+}$ GFAP-CreER and NFIA ${ }^{f / f l}$ GFAPCreER mice using previously described procedures $(25,53)$. Following an additional 7 days of culturing, astrocytes were seeded on opposing sides of Transwell inserts $(0.4-\mu \mathrm{m}$ pore size, 12 -well; Corning) using methods previously described $(25,53)$. Briefly, astrocytes were seeded on the abluminal side of the poly-D-lysine-coated insert at a density of $3 \times 10^{4}$ cells per filter and allowed to adhere for 2 hours before being inverted back into DMEM F12 containing 10\% FBS and 1\% 33) penicillin streptomycin (PS) media. After allowing the astrocytes to grow for 48 hours, the inserts were plated with immortalized mouse endothelial bEnd 3 cells (American Type Culture Collection) at a density of $6.6 \times 10^{4}$ cells per filter. TEER measurements were then made 24 hours after bEnd 3 seeding using an EVOM resistance meter (World Precision Instruments) with Stx2 electrodes. TEER resistance was measured in ohms and normalized to total DAPI ${ }^{+}$ nuclei on the abluminal side of the membrane (astrocytes) after subtracting resistance from blank Transwell inserts.

Evans blue quantification. Evans blue was quantified as previously described (54). Briefly, 3-month-old mice were injected i.p. with a filtered $2 \%$ Evans blue solution in PBS $(150 \mu \mathrm{L} / 10$ g body weight). Twentyfour hours later, mice were perfused with $1 \times$ PBS, and spinal cords or cortices were harvested, weighed, and incubated with $500 \mu \mathrm{L}$ formamide for 24 hours at $65^{\circ} \mathrm{C}$. Subsequently, the samples were centrifuged for 30 minutes at 13,200 rpm, and absorbance was read at $600 \mathrm{~nm}$. In parallel, a standard curve of Evans blue dye was performed to calculate the amount of Evans in $\mathrm{ng} / \mu \mathrm{l} / \mathrm{mg}$ tissue.

ChIP. Primary cultures of astrocytes from mouse cortex (from P1-P3 mice) were used for ChIP assays. Harvested cells were washed with PBS buffer and crosslinked with freshly prepared $1 \%$ formaldehyde for 10 minutes at room temperature with rocking. After washing with PBS (containing $1 \mathrm{mM}$ PMSF), the cells were collected by scraping. The cell pellets were further washed with PBS, buffer 1 (10 mM EDTA, 0.5 mM EGTA, 0.25\% Triton X-100, 10 mM HEPES, pH 6.5), and buffer 2 (200 mM NaCl, 1 mM EDTA, 0.5 mM EGTA, 10 $\mathrm{mM}$ HEPES, $\mathrm{pH} 6.5)$. The pellets were frozen at $-80^{\circ} \mathrm{C}$ at this stage or resuspended in lysis buffer (0.5\% SDS, 5 mM EDTA, $25 \mathrm{mM}$ Tris, pH 8 , Roche protease inhibitor cocktail). The suspension was sonicated at $4^{\circ} \mathrm{C}$ in a Branson Sonifier 450 . The supernatant was collected and diluted 5-fold with dilution buffer (20 mM Tris, pH 8, $150 \mathrm{mM} \mathrm{NaCl}$, 2 mM EDTA, 1\% Triton X-100, Roche protease inhibitor cocktail), followed by a preclearing-step mouse IgG (Santa Cruz Biotechnology, sc-2025) and protein A beads (Invitrogen, Thermo Fisher Scientific) at $4^{\circ} \mathrm{C}$ for 1 hour with shaking. After centrifugation to collect the cell lysate, NFIA antibody (in-house preparation, $10 \mu \mathrm{g}$ ) and rabbit IgG (Santa Cruz Biotechnology, sc-2027) were added to separate immunoprecipitation tubes at $4^{\circ} \mathrm{C}$ overnight with shaking. A further incubation with protein $\mathrm{A}$ beads at $4^{\circ} \mathrm{C}$ for 6 hours was done, followed by collection of the beads and washing them 4 times for 5 minutes each time at room temperature with shaking, with cold TSE1 buffer(0.1\% SDS, 1\% Triton X-100, 2 mM EDTA, 150 mM NaCl, 20 mM Tris, pH 8), cold TSE2 buffer (0.1\% SDS, 1\% Triton X-100, 2 mM EDTA, $500 \mathrm{mM} \mathrm{NaCl}, 20 \mathrm{mM}$ Tris, pH 8), LiCl buffer (0.25M LiCl, $1 \%$ NP-40, 1\% Na deoxycholate, 1 mM EDTA, 10 mM Tris, pH 8), and TE buffer (10 mM Tris, pH 8, 1 mM EDTA). The TE buffer wash was repeated twice, and elution buffer (1\% SDS, $0.1 \mathrm{M} \mathrm{NaHCO}_{3}$ ) was added to the beads and vortexed, followed by rocking at room temperature for 10 minutes. The elution was repeated, and the supernatant was collected and incubated at $65^{\circ} \mathrm{C}$ overnight, followed by a 2-hour incubation at $42^{\circ} \mathrm{C}$ with Proteinase K (Promega, $0.05 \mathrm{mg}$ / $\mathrm{mL}$ ). Genomic fragments were purified with phenol-chloroform extraction, and the DNA pellet was resuspended in water. The ChIP DNA was analyzed by PCR using primers specific to the Thbs 4 promoter (forward: 5'CACCGCATTCCATTCCGTCT; reverse: 5'-CCGCGCTTTATGGTCCAG).

Imaging of fluorescent and colorimetric staining. Imaging was performed using Zeiss Imager M2 and a Z1 fluorescence microscope equipped with a Zeiss AxioCam camera. Analysis was performed using AxioVision and Image J software (NIH).

Statistics. $P$ values were determined using a 2-tailed Student's $t$ test. A 1-way ANOVA was used to analyze the expression of markers to determine the differences between group means, followed by Tukey's test to compare individual means. All statistical tests were 2 sided. No statistical methods were used to predetermine sample sizes, but our sample sizes were similar to those reported in previous publications $(8,11)$. Data distribution was assumed to be normal, but this was not formally tested. Randomization of animal studies was used in the data analysis. Data collection and analysis were not performed in a blinded manner with regard to the experimental conditions. No animal or data points were excluded from the analyses. $P$ values of greater than 0.05 were considered statistically significant.

Study approval. All housing and breeding procedures were approved by the IACUC of the Baylor College of Medicine and conformed with the US Public Health Service Policy on Human Care and Use of Laboratory Animals. All animal procedures were performed in accordance with protocols approved by the IACUC of the Baylor College of Medicine. Human HIE tissue was collected in accordance with guidelines established by the Baylor College of Medicine (H35483). Adult brain MS tissue and healthy brain tissue were acquired through collaboration with the Rocky Mountain Multiple Sclerosis Society.

\section{Author contributions}

DL, TWH, HKL, CTK, and BD conceived the project and designed the experiments. DL, TWH, NABH, YSH, DS, JO, JCC, and HKL performed the experiments. CAM, CTK, BRA, and SMG provided essential reagents. CAM performed all histopathological analyses. $\mathrm{DL}$ and BD wrote the manuscript. DL and TWH are listed as co-first authors on the basis of equitable contributions to this manuscript.

\section{Acknowledgments}

This work was supported by grants from the National Multiple Sclerosis Society (RG-1501-02756, to BD, and FG-1607-25417, to $\mathrm{TH}$ ); the NIH (NS096096, to BD and CTK, and NSO71153, to BD); and the National Heart, Lung, and Blood Institute (NHLBI), NIH (T32-HL902332, to DL). Human multiple sclerosis tissue was provided by the tissue bank at the Rocky Mountain MS Center of the University of Colorado.

Address correspondence to: Benjamin Deneen, Center for Cell and Gene Therapy, Department of Neurosurgery, One Baylor Plaza, N1120.06, Houston, Texas 77030, USA. Phone: 713.798.7897; Email: deneen@bcm.edu. 
1. Sofroniew MV, Vinters HV. Astrocytes: biology and pathology. Acta Neuropathol. 2010;119(1):7-35.

2. Renault-Mihara F, Okada S, Shibata S, Nakamura M, Toyama Y, Okano H. Spinal cord injury: emerging beneficial role of reactive astrocytes' migration. Int J Biochem Cell Biol. 2008;40(9):1649-1653.

3. Voskuhl RR, et al. Reactive astrocytes form scarlike perivascular barriers to leukocytes during adaptive immune inflammation of the CNS. J Neurosci. 2009;29(37):11511-11522.

4. Liddelow SA, et al. Neurotoxic reactive astrocytes are induced by activated microglia. Nature. 2017;541(7638):481-487.

5. Silver J, Miller JH. Regeneration beyond the glial scar. Nat Rev Neurosci. 2004;5(2):146-156.

6. Pekny M, Pekna M. Astrocyte reactivity and reactive astrogliosis: costs and benefits. Physiol Rev. 2014;94(4):1077-1098.

7. Liddelow SA, Barres BA. Reactive astrocytes: production, function, and therapeutic potential. Immunity. 2017;46(6):957-967.

8. John Lin CC, et al. Identification of diverse astrocyte populations and their malignant analogs. Nat Neurosci. 2017;20(3):396-405.

9. Sofroniew MV. Molecular dissection of reactive astrogliosis and glial scar formation. Trends Neurosci. 2009;32(12):638-647.

10. Anderson MA, Ao Y, Sofroniew MV. Heterogeneity of reactive astrocytes. Neurosci Lett. 2014;565:23-29.

11. Chaboub LS, et al. Temporal profiling of astrocyte precursors reveals parallel roles for Asef during development and after injury. J Neurosci. 2016;36(47):11904-11917.

12. Herrmann JE, et al. STAT3 is a critical regulator of astrogliosis and scar formation after spinal cord injury. J Neurosci. 2008;28(28):7231-7243.

13. Nobuta $\mathrm{H}$, et al. STAT3-mediated astrogliosis protects myelin development in neonatal brain injury. Ann Neurol. 2012;72(5):750-765.

14. Deneen B, Ho R, Lukaszewicz A, Hochstim CJ, Gronostajski RM, Anderson DJ. The transcription factor NFIA controls the onset of gliogenesis in the developing spinal cord. Neuron. 2006;52(6):953-968.

15. Kang P, et al. Sox9 and NFIA coordinate a transcriptional regulatory cascade during the initiation of gliogenesis. Neuron. 2012;74(1):79-94.

16. Glasgow SM, et al. Mutual antagonism between Sox10 and NFIA regulates diversification of glial lineages and glioma subtypes. Nat Neurosci. 2014;17(10):1322-1329.

17. Glasgow SM, et al. Glia-specific enhancers and chromatin structure regulate NFIA expression and glioma tumorigenesis. Nat Neurosci. 2017;20(11):1520-1528.

18. Fancy SP, Glasgow SM, Finley M, Rowitch DH, Deneen B. Evidence that nuclear factor IA inhibits repair after white matter injury. Ann Neurol. 2012;72(2):224-233.

19. Benner EJ, et al. Protective astrogenesis from the SVZ niche after injury is controlled by Notch modulator Thbs4. Nature. 2013;497(7449):369-373.

20. Labat-gest V, Tomasi S. Photothrombotic isch- emia: a minimally invasive and reproducible photochemical cortical lesion model for mouse stroke studies. JVis Exp. 2013;(76)e50370.

21. Harrington EP, Zhao C, Fancy SP, Kaing S, Franklin RJ, Rowitch DH. Oligodendrocyte PTEN is required for myelin and axonal integrity, not remyelination. Ann Neurol. 2010;68(5):703-716.

22. Fancy SP, et al. Axin 2 as regulatory and therapeutic target in newborn brain injury and remyelination. Nat Neurosci. 2011;14(8):1009-1016.

23. Kremer D, Aktas O, Hartung HP, Küry P. The complex world of oligodendroglial differentiation inhibitors. Ann Neurol. 2011;69(4):602-618.

24. Obermeier B, Daneman R, Ransohoff RM. Development, maintenance and disruption of the bloodbrain barrier. Nat Med. 2013;19(12):1584-1596.

25. Li G, et al. Permeability of endothelial and astrocyte cocultures: in vitro blood-brain barrier models for drug delivery studies. Ann Biomed Eng. 2010;38(8):2499-2511.

26. Sun W, et al. SOX9 is an astrocyte-specific Nnuclear marker in the adult brain outside the neurogenic regions. J Neurosci. 2017;37(17):4493-4507.

27. Rankin KA, et al. Selective estrogen receptor modulators enhance $\mathrm{CNS}$ remyelination independent of estrogen receptors. J Neurosci. 2019;39(12):2184-2194

28. Faiz M, Sachewsky N, Gascón S, Bang KW, Morshead CM, Nagy A. Adult neural stem cells from the subventricular zone give rise to reactive astrocytes in the cortex after stroke. Cell Stem Cell. 2015;17(5):624-634.

29. Sun Y, et al. Neurogenin promotes neurogenesis and inhibits glial differentiation by independent mechanisms. Cell. 2001;104(3):365-376.

30. He F, et al. A positive autoregulatory loop of JakSTAT signaling controls the onset of astrogliogenesis. Nat Neurosci. 2005;8(5):616-625.

31. Koblar SA, et al. Neural precursor differentiation into astrocytes requires signaling through the leukemia inhibitory factor receptor. Proc Natl Acad Sci U S A. 1998;95(6):3178-3181.

32. Hong S, Song MR. STAT3 but not STAT1 is required for astrocyte differentiation. PLOS ONE 2014;9(1):e86851.

33. Lütolf S, Radtke F, Aguet M, Suter U, Taylor V. Notch1 is required for neuronal and glial differentiation in the cerebellum. Development. 2002;129(2):373-385.

34. Shimada IS, Borders A, Aronshtam A, Spees JL. Proliferating reactive astrocytes are regulated by Notch-1 in the peri-infarct area after stroke. Stroke. 2011;42(11):3231-3237.

35. Hammond TR, et al. Astrocyte-derived endothelin-1 inhibits remyelination through notch activation. Neuron. 2014;81(3):588-602.

36. Namihira M, et al. Committed neuronal precursors confer astrocytic potential on residual neural precursor cells. Dev Cell. 2009;16(2):245-255.

37. Burda JE, Sofroniew MV. Reactive gliosis and the multicellular response to CNS damage and disease. Neuron. 2014;81(2):229-248.

38. Eddleston M, Mucke L. Molecular profile of reactive astrocytes--implications for their role in neu- rologic disease. Neuroscience. 1993;54(1):15-36.

39. Daginakatte GC, et al. Expression profiling identifies a molecular signature of reactive astrocytes stimulated by cyclic AMP or proinflammatory cytokines. Exp Neurol. 2008;210(1):261-267.

40. Yeh TH, Lee DY, Gianino SM, Gutmann DH. Microarray analyses reveal regional astrocyte heterogeneity with implications for neurofibromatosis type 1 (NF1)-regulated glial proliferation. Glia. 2009;57(11):1239-1249.

41. Chai $\mathrm{H}$, et al. Neural circuit-specialized astrocytes: transcriptomic, proteomic, morphological, and runctional evidence. Neuron. 2017;95(3):531-549.e9.

42. Morel L, et al. Molecular and functional properties of regional astrocytes in the adult brain. JNeurosci. 2017;37(36):8706-8717.

43. Molofsky AV, et al. Astrocyte-encoded positional cues maintain sensorimotor circuit integrity. Nature. 2014;509(7499):189-194.

44. Tsai HH, et al. Regional astrocyte allocation regulates CNS synaptogenesis and repair. Science. 2012;337(6092):358-362.

45. Hochstim C, Deneen B, Lukaszewicz A, Zhou $\mathrm{Q}$, Anderson DJ. Identification of positionally distinct astrocyte subtypes whose identities are specified by a homeodomain code. Cell. 2008;133(3):510-522.

46. Rowitch DH, Kriegstein AR. Developmental genetics of vertebrate glial-cell specification. Nature. 2010;468(7321):214-222.

47. Burrows RC, Wancio D, Levitt P, Lillien L. Response diversity and the timing of progenitor cell maturation are regulated by developmental changes in EGFR expression in the cortex. Neuron. 1997;19(2):251-267.

48. Aguirre A, Gallo V. Postnatal neurogenesis and gliogenesis in the olfactory bulb from NG2expressing progenitors of the subventricular zone. J Neurosci. 2004;24(46):10530-10541.

49. Aguirre A, Dupree JL, Mangin JM, Gallo V. A functional role for EGFR signaling in myelination and remyelination. Nat Neurosci. 2007;10(8):990-1002.

50. Glasgow SM, et al. The miR-223/nuclear factor I-A axis regulates glial precursor proliferation and tumorigenesis in the CNS. JNeurosci. 2013;33(33):13560-13568.

51. Buffo A, et al. Origin and progeny of reactive gliosis: A source of multipotent cells in the injured brain. Proc Natl Acad Sci U S A. 2008;105(9):3581-3586.

52. Lein ES, et al. Genome-wide atlas of gene expression in the adult mouse brain. Nature. 2007;445(7124):168-176.

53. Helms HC, et al. In vitro models of the bloodbrain barrier: an overview of commonly used brain endothelial cell culture models and guidelines for their use. JCereb Blood Flow Metab. 2016;36(5):862-890.

54. Menezes MJ, McClenahan FK, Leiton CV, Aranmolate A, Shan X, Colognato H. The extracellular matrix protein laminin $\alpha 2$ regulates the maturation and function of the blood-brain barrier. JNeurosci. 2014;34(46):15260-15280. 